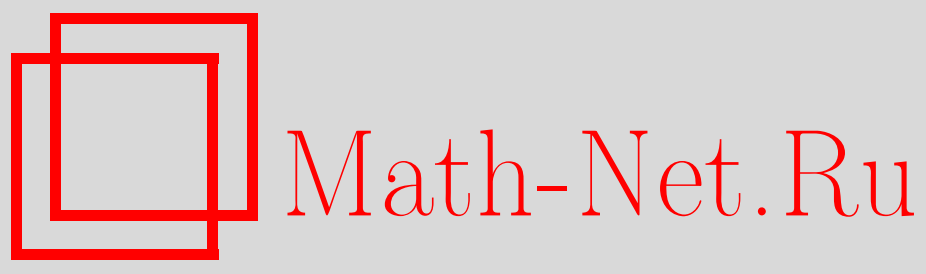

А. Е. Миронов, Коммутирующие дифференциальные операторы ранга 2, отвечающие кривой рода 2, Функи. анализ и его прил., 2005, том 39, выпуск $3,91-94$

DOI: https://doi.org/10.4213/faa80

Использование Общероссийского математического портала Math-Net.Ru подразумевает, что вы прочитали и согласны с пользовательским соглашением

http://www.mathnet.ru/rus/agreement

Параметры загрузки:

IP : 54.224 .135 .184

26 апреля 2023 г., 14:32:09

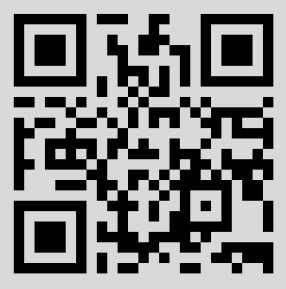


44, 571-636 (1980). 8. Kirchberg E. Invent. Math., 112, 449-489 (1993). 9. Manuilov V. M., Thomsen K. J. Funct. Anal., 213, 154-175 (2004). 10. Manuilov V. M., Thomsen K. Proc. London Math. Soc., 88, 455-478 (2004). 11. Manuilov V. M., Thomsen K. K-Theory, 32, 101-138 (2004).

Московский государственный университет, механико-математический факультет,

Поступило в редакцию e-mail: manuilov@mech.math.msu.su 30 января 2004 г.

Institut for Matematiske Fag, Ny Munkegade, Denmark e-mail: matkt@imf.au.dk

УДК 517.43

\title{
Коммутирующие дифференциальные операторы ранга 2, отвечающие кривой рода $2^{*}$
}

\author{
(c) 2005. А. Е. Миронов
}

1. Введение. В данной статье построены некоторые обыкновенные формально самосопряженные коммутирующие дифференциальные операторы ранга 2 , отвечающие кривой рода 2 , причем функция Бейкера-Ахиезера этих операторов имеет существенную особенность в точке Вейерштрасса.

Обозначим через $L_{1}$ и $L_{2}$ дифференциальные операторы

$$
L_{1}=\partial_{x}^{n}+\sum_{i=0}^{n-2} u_{i}(x) \partial_{x}^{i}, \quad L_{2}=\partial_{x}^{m}+\sum_{i=0}^{m-2} v_{i}(x) \partial_{x}^{i} .
$$

Напомним, что по лемме Берчналла-Чаунди, если $L_{1} L_{2}=L_{2} L_{1}$, то существует полином $Q(z, w)$ от двух коммутирующих переменных, такой, что $Q\left(L_{1}, L_{2}\right)=0$. Обозначим через Г кривую, заданную в плоскости $(z, w)$ уравнением $Q(z, w)=0$. Для $P \in \Gamma$ в общем положении существует совместная собственная функция $\psi(x, P)$ (функция Бейкера-Ахиезера) операторов $L_{1}$ и $L_{2}$, такая, что $L_{1} \psi=z \psi$, $L_{2} \psi=w \psi$. Размерность $l$ пространства таких функций, отвечающих $P$, называется рангом пары $L_{1}, L_{2}$. Для операторов ранга 1 функция $\psi$ выражается явным образом через тэта-функцию многообразия Якоби кривой Г, и в этом случае коэффициенты операторов $L_{1}$ и $L_{2}$ легко найти [1]. Случай $l>1$ существенно сложнее. Задача отыскания операторов ранга $l>1$ в общем случае до сих пор не решена. Новиков и Кричевер [2] нашли операторы ранга 2, отвечающие кривой рода 1. Мохов [3] нашел операторы ранга 3 рода 1.

Основной результат этой работы заключается в следующем. Пусть $l=2$ и кривая Г рода 2 является гладким пополнением бесконечно удаленной точкой $\infty$ кривой, заданной в $\mathbb{C}^{2}$ уравнением

$$
w^{2}=F(z)=z^{5}+c_{3} z^{3}+c_{2} z^{2}+c_{1} z+c_{0} .
$$

Кривая $Г$ допускает голоморфную инволюцию $\sigma: \Gamma \rightarrow \Gamma, \sigma(z, w)=(z,-w)$, которая имеет 6 неподвижных точек $\infty,\left(z_{i}, 0\right)$, где $z_{i}$ - точки ветвления (корни уравнения $F(z)=0)$.

*Работа поддержана грантами РФФИ (03-01-00403 и 03-01-06482) и грантом НШ2185.2003 .1 
Существуют функции $\chi_{0}(x, P), \chi_{1}(x, P)$ (см. [4]), такие, что $\psi_{i}^{\prime \prime}=\chi_{0} \psi_{i}+$ $\chi_{1} \psi_{i}^{\prime}$, где $\psi_{i}$ - функция Бейкера-Ахиезера, $i=0,1$. Коэффициенты операторов $L_{1}$ и $L_{2}$ находятся по $\chi_{0}, \chi_{1}$. Мы рассматриваем случай, когда $\sigma \chi_{1}=\chi_{1}$. Справедлива следующая

Теорема. Имеют место равенства

$$
\begin{gathered}
\chi_{1}=-\frac{\gamma^{\prime}}{z-\gamma}-\frac{\gamma^{\prime}}{z-(\gamma+c)}, \\
\chi_{0}=-\frac{1}{2} \frac{H_{1} \gamma^{\prime}}{z-\gamma}-\frac{1}{2} \frac{H_{2} \gamma^{\prime}}{z-(\gamma+c)}+\frac{\kappa}{2}+\frac{1}{2} \frac{w}{(z-\gamma)(z-(\gamma+c))},
\end{gathered}
$$

əде

$$
\begin{gathered}
\kappa=\frac{F(\gamma)-4 \gamma^{\prime 4}+8 c \gamma^{\prime 2} \gamma^{\prime \prime}-c^{2} \gamma^{\prime \prime 2}+2 c^{2} \gamma^{\prime} \gamma^{\prime \prime \prime}}{2 c^{2} \gamma^{\prime 2}}, \\
\gamma^{-1}(y)=\int\left(\frac{4}{3 R(y)}\right)^{1 / 4} d y
\end{gathered}
$$

$R(y)=y^{5}+\frac{5}{2} c y^{4}+\frac{1}{3}\left(10 c^{2}+3 c_{3}\right) y^{3}+\frac{1}{2}\left(5 c_{3}+2 c_{2}+3 c c_{3}\right) y^{2}+\left(c^{4}+c_{1}+c c_{2}+c^{2} c_{3}\right) y+\delta$, $y=\gamma(x), \delta, c \in \mathbb{C}$. Функиии $H_{i}(x)$ выражсаются через $\gamma(x)$ по формулам (8)(10) (см. ниже).

СледСтвиЕ. Oператор, отвечающий функиии z, имеет вид

$$
L=L^{*}=\partial_{x}^{4}-\kappa \partial_{x}^{2}-\kappa^{\prime} \partial_{x}+\frac{\kappa^{2}}{4}-\frac{\kappa^{\prime \prime}}{2}-\gamma-\frac{c}{2} .
$$

Укажем пример. Пусть кривая Г задается уравнением $w^{2}=z^{5}-\frac{10}{3} z^{3}+\frac{7}{3} z$ и $c=2, \delta=1, \gamma=1024 / x^{4}-1$. Тогда $L$ имеет вид

$$
\partial_{x}^{4}+\left(\frac{x^{6}}{49152}-\frac{425}{6 x^{2}}\right) \partial_{x}^{2}+\left(\frac{x^{5}}{8192}+\frac{425}{3 x^{3}}\right) \partial_{x}+\frac{x^{12}}{9663676416}-\frac{245 x^{4}}{589824}+\frac{2569}{144 x^{4}} .
$$

Оператор десятого порядка, отвечающий функции $w$, мы здесь не приводим из-за его громоздкости.

2. Доказательство теоремы. Кричевер [4] доказал, что в случае кривой рода 2 и $l=2$ функции $\chi_{j}(x, P)$ являются мероморфными на $\Gamma$ с четырьмя полюсами $P_{1}(x), \ldots, P_{4}(x)$. В окрестности $\infty$ функции $\chi_{j}$ имеют вид

$$
\chi_{0}(x, P)=k+g_{0}(x)+O\left(k^{-1}\right), \quad \chi_{1}(x, P)=O\left(k^{-1}\right),
$$

где $k^{-1}$ - локальный параметр около $\infty$, а в окрестности полюса $P_{i}(x)-$ вид

$$
\begin{aligned}
& \chi_{0}(x, P)=\frac{-\alpha_{i}(x) \gamma_{i}^{\prime}(x)}{k-\gamma_{i}(x)}+d_{i 0}(x)+O\left(k-\gamma_{i}(x)\right), \\
& \chi_{1}(x, P)=\frac{-\gamma_{i}^{\prime}(x)}{k-\gamma_{i}(x)}+d_{i 1}(x)+O\left(k-\gamma_{i}(x)\right),
\end{aligned}
$$

где $k-\gamma_{i}(x)$ - локальный параметр около $P_{i}(x)$, причем

$$
d_{i 0}(x)=\alpha_{i}^{2}(x)+\alpha_{i}(x) d_{i 1}(x)-\alpha_{i}^{\prime}(x) .
$$


Выберем в окрестности полюса $P_{i}(x)$ локальный параметр $z-\gamma_{i}(x)$, а в окрестности $\infty$ локальный параметр $k^{-1}=2 / \sqrt{z}$. Пусть $\sigma \chi_{1}=\chi_{1}$. Тогда можно считать, что $\sigma P_{1}(x)=P_{3}(x)$ и $\sigma P_{2}(x)=P_{4}(x)$, а следовательно, $\gamma_{1}=\gamma_{3}, \gamma_{2}=\gamma_{4}$, $d_{11}=d_{31}, d_{21}=d_{41}$. Таким образом, в силу формул (2) и (4) имеем

$$
\chi_{1}=\frac{1}{2}\left(\chi_{1}+\sigma \chi_{1}\right)=\frac{-\gamma_{1}^{\prime}}{z-\gamma_{1}}+\frac{-\gamma_{2}^{\prime}}{z-\gamma_{2}}, \quad d_{11}=\frac{-\gamma_{2}^{\prime}}{\gamma_{1}-\gamma_{2}}, \quad d_{21}=\frac{-\gamma_{1}^{\prime}}{\gamma_{2}-\gamma_{1}} .
$$

Так как функция $\chi_{0}+\sigma \chi_{0}$ не зависит от $w$, то $\chi_{0}+\sigma \chi_{0}$ не может иметь полюс первого порядка в $\infty$; следовательно, из формул (2) и (3) получаем

$$
\begin{aligned}
\chi_{0}+\sigma \chi_{0} & =-\frac{H_{1} \gamma_{1}^{\prime}}{z-\gamma_{1}}-\frac{H_{2} \gamma_{2}^{\prime}}{z-\gamma_{2}}+\kappa, \\
\left(\chi_{0}-\sigma \chi_{0}\right)^{2} & =\frac{F_{1} \gamma_{1}^{\prime 2}}{\left(z-\gamma_{1}\right)^{2}}+\frac{2 G_{1} \gamma_{1}^{\prime}}{z-\gamma_{1}}+\frac{F_{2} \gamma_{2}^{\prime 2}}{\left(z-\gamma_{2}\right)^{2}}+\frac{2 G_{2} \gamma_{2}^{\prime}}{z-\gamma_{2}}+z+\kappa_{1},
\end{aligned}
$$

где $\kappa(x)$ и $\kappa_{1}(x)$ - некоторые функции, $H_{1}=\alpha_{1}+\alpha_{3}, H_{2}=\alpha_{2}+\alpha_{4}, F_{1}=$ $\left(\alpha_{3}-\alpha_{1}\right)^{2}, F_{2}=\left(\alpha_{4}-\alpha_{2}\right)^{2}, G_{1}=\left(d_{10}-d_{30}\right)\left(\alpha_{3}-\alpha_{1}\right), G_{2}=\left(d_{20}-d_{40}\right)\left(\alpha_{4}-\alpha_{2}\right)$. Согласно равенству (6),

$$
\begin{gathered}
d_{10}+d_{30}=-\frac{H_{2} \gamma_{2}^{\prime}}{\gamma_{1}-\gamma_{2}}+\kappa, \quad d_{20}+d_{40}=-\frac{H_{1} \gamma_{1}^{\prime}}{\gamma_{2}-\gamma_{1}}+\kappa, \\
d_{10}+d_{30}-\left(d_{20}+d_{40}\right)=\frac{H_{1} \gamma_{1}^{\prime}}{\gamma_{2}-\gamma_{1}}-\frac{H_{2} \gamma_{2}^{\prime}}{\gamma_{1}-\gamma_{2}} .
\end{gathered}
$$

Функция $\left(\chi_{0}-\sigma \chi_{0}\right)^{2}$ имеет нули в пяти точках ветвления $\left(z_{i}, 0\right)$, а следовательно, $\left(\chi_{0}-\sigma \chi_{0}\right)^{2}\left(z-\gamma_{1}\right)^{2}\left(z-\gamma_{2}\right)^{2}=F(z)$, откуда вытекает, что

$$
\begin{gathered}
F_{1}=\frac{F\left(\gamma_{1}\right)}{\left(\gamma_{1}-\gamma_{2}\right)^{2} \gamma_{1}^{\prime 2}}, \quad F_{2}=\frac{F\left(\gamma_{2}\right)}{\left(\gamma_{1}-\gamma_{2}\right)^{2} \gamma_{2}^{\prime 2}} \\
G_{1}=\frac{2 c_{0}+c_{1} \gamma_{1}-c_{3} \gamma_{1}^{3}-3 \gamma_{1}^{5}+\gamma_{2}\left(c_{1}+2 c_{2} \gamma_{1}+3 c_{3} \gamma_{1}^{2}+5 \gamma_{1}^{4}\right)}{2\left(\gamma_{2}-\gamma_{1}\right)^{3} \gamma_{1}^{\prime}} \\
G_{2}=\frac{2 c_{0}+c_{1} \gamma_{2}-c_{3} \gamma_{2}^{2}-3 \gamma_{2}^{5}+\gamma_{1}\left(c_{1}+2 c_{2} \gamma_{2}+3 c_{3} \gamma_{2}^{2}+5 \gamma_{2}^{4}\right)}{2\left(\gamma_{1}-\gamma_{2}\right)^{3} \gamma_{2}^{\prime}}
\end{gathered}
$$

Выразим $H_{1}$ и $H_{2}$ через $\gamma_{1}$ и $\gamma_{2}$. В силу формулы (5)

$$
\begin{aligned}
& d_{10}-d_{30}=\alpha_{1}^{2}-\alpha_{3}^{2}+d_{11}\left(\alpha_{1}-\alpha_{3}\right)-\left(\alpha_{1}^{\prime}-\alpha_{3}^{\prime}\right), \\
& d_{20}-d_{40}=\alpha_{2}^{2}-\alpha_{4}^{2}+d_{21}\left(\alpha_{2}-\alpha_{4}\right)-\left(\alpha_{2}^{\prime}-\alpha_{4}^{\prime}\right) .
\end{aligned}
$$

Умножим эти равенства соответственно на $\alpha_{3}-\alpha_{1}$ и на $\alpha_{4}-\alpha_{2}$ и получим

$$
\begin{aligned}
& H_{1}=\frac{\frac{1}{2} F_{1}^{\prime}-d_{11} F_{1}-G_{1}}{F_{1}}=\frac{2 \gamma_{1}^{\prime} \gamma_{2}^{\prime}+\left(\gamma_{2}-\gamma_{1}\right) \gamma_{1}^{\prime \prime}}{\left(\gamma_{1}-\gamma_{2}\right) \gamma_{1}^{\prime}} \\
& H_{2}=\frac{\frac{1}{2} F_{2}^{\prime}-d_{21} F_{2}-G_{2}}{F_{2}}=\frac{-2 \gamma_{1}^{\prime} \gamma_{2}^{\prime}+\left(\gamma_{2}-\gamma_{1}\right) \gamma_{2}^{\prime \prime}}{\left(\gamma_{1}-\gamma_{2}\right) \gamma_{2}^{\prime}}
\end{aligned}
$$


Далее,

$$
\begin{aligned}
& \alpha_{1}^{2}+\alpha_{3}^{2}=\frac{1}{2}\left(\alpha_{1}+\alpha_{3}\right)^{2}+\frac{1}{2}\left(\alpha_{1}-\alpha_{3}\right)^{2}=\frac{1}{2} H_{1}^{2}+\frac{1}{2} F_{1}, \\
& \alpha_{2}^{2}+\alpha_{4}^{2}=\frac{1}{2}\left(\alpha_{2}+\alpha_{4}\right)^{2}+\frac{1}{2}\left(\alpha_{2}-\alpha_{4}\right)^{2}=\frac{1}{2} H_{2}^{2}+\frac{1}{2} F_{2} .
\end{aligned}
$$

Выразим $d_{10}+d_{30}$ и $d_{20}+d_{40}$ через $\gamma_{1}$ и $\gamma_{2}$. Из равенства (5) выводим, что

$$
d_{10}+d_{30}=\frac{H_{1}^{2}}{2}+\frac{F_{1}}{2}-H_{1}^{\prime}+d_{11} H_{1}, \quad d_{20}+d_{40}=\frac{H_{2}^{2}}{2}+\frac{F_{2}}{2}-H_{2}^{\prime}+d_{21} H_{2} .
$$

Подставим полученные выражения для $H_{1}, H_{2}, d_{10}+d_{30}$ и $d_{20}+d_{40}$ в (7). Получим уравнение на $\gamma_{1}$ и $\gamma_{2}$ :

$$
\begin{aligned}
4 \gamma_{1}^{\prime 4} & \gamma_{2}^{\prime 2}+\gamma_{2}^{\prime 2}\left(c_{0}+c_{3} \gamma_{1}^{3}+\gamma_{1}^{5}-\gamma_{2}^{2} \gamma_{1}^{\prime \prime 2}+\gamma_{1}^{2}\left(c_{2}-\gamma_{1}^{\prime \prime 2}\right)+\gamma_{1}\left(c_{1}+2 \gamma_{2} \gamma_{1}^{\prime \prime 2}\right)\right) \\
& +2\left(\gamma_{2}-\gamma_{1}\right) \gamma_{1}^{\prime 3} \gamma_{2}^{\prime} \gamma_{2}^{\prime \prime}+2\left(\gamma_{1}-\gamma_{2}\right) \gamma_{1}^{\prime} \gamma_{2}^{\prime 2}\left(\left(\gamma_{1}-\gamma_{2}\right) \gamma_{1}^{\prime \prime \prime}-\gamma_{2}^{\prime} \gamma_{1}^{\prime \prime}\right) \\
& -\gamma_{1}^{\prime 2}\left(c_{0}+c_{3} \gamma_{2}^{3}+\gamma_{2}^{5}+4 \gamma_{2}^{\prime 4}-\gamma_{1}^{2} \gamma_{2}^{\prime \prime 2}+6 \gamma_{1} \gamma_{2}^{\prime 2}\left(\gamma_{1}^{\prime \prime}+\gamma_{2}^{\prime \prime}\right)+2 \gamma_{1}^{2} \gamma_{2}^{\prime} \gamma_{2}^{\prime \prime \prime}\right. \\
& \left.+\gamma_{2}^{2}\left(c_{2}-\gamma_{2}^{\prime \prime 2}+2 \gamma_{2}^{\prime} \gamma_{2}^{\prime \prime \prime}\right)+\gamma_{2}\left(c_{1}+2 \gamma_{1} \gamma_{2}^{\prime \prime 2}-6 \gamma_{2}^{\prime 2}\left(\gamma_{1}^{\prime \prime}+\gamma_{2}^{\prime \prime}\right)-4 \gamma_{1} \gamma_{2}^{\prime} \gamma_{2}^{\prime \prime \prime}\right)\right)=0 .
\end{aligned}
$$

При

это уравнение принимает вид

$$
\gamma_{1}=\gamma, \quad \gamma_{2}=\gamma+c
$$

$\left(c^{4}+c_{1}+c c_{2}+c^{2} c_{3}\right)+\left(5 c_{3}+2 c_{2}+3 c c_{3}\right) \gamma+\left(10 c^{2}+3 c_{3}\right) \gamma^{2}+10 c \gamma^{3}+5 \gamma^{4}-16 \gamma^{\prime 2} \gamma^{\prime \prime}=0$ и интегрируется в квадратурах (см. (1)). Теорема 1 доказана.

\section{ЛитеРАТУРА}

1. Кричевер И. М. Функц. анализ и его прил., 11, вып. 1, 15-31 (1977). 2. Кричевер И. М., Новиков С. П. Функц. анализ и его прил., 12, вып. 4, 41-52 (1978). 3. Мохов О. И. Изв. АН СССР, сер. матем., 53, №6, 1291-1314 (1989). 4. Кричевер И. М. Функц. анализ и его прил., 12, вып. 4, 41-52 (1978).

Институт математики им. С. Л. Соболева

СО РАН, Новосибирск

Поступило в редакцию e-mail: mironov@math.nsc.ru 